\title{
Nonlinear force-free modeling of the corona in spherical
}

\section{coordinates}

\section{S.A. Gilchrist ${ }^{1}$ - M.S. Wheatland ${ }^{1}$}

(C) Springer $\bullet \bullet \bullet \bullet$

\begin{abstract}
We present a code for solving the nonlinear force-free equations in spherical polar geometry, with the motivation of modeling the magnetic field in the corona. The code is an implementation of the Grad-Rubin method. Our method is applicable to a spherical domain of arbitrary angular size. The implementation is based on a global spectral representation for the magnetic field which makes no explicit assumptions about the form of the magnetic field at the transverse boundaries of the domain. We apply the code to a bipolar test case with analytic boundary conditions, and we demonstrate the convergence of the Grad-Rubin method, and the self-consistency of the resulting numerical solution.
\end{abstract}

\footnotetext{
${ }^{1}$ Sydney Institute for Astronomy, School of Physics, The University of Sydney, NSW 2006, Australia
} 


\section{Introduction}

Basic properties of the solar coronal magnetic field such as its strength, direction, and three-dimensional structure, cannot be presently determined by observation, and this motivates modeling of the coronal magnetic field. In the corona above active regions the pressure and gravity forces are too small to balance the magnetic (Lorentz) force (Metcalf et al., 1995; Garv, 2001) so it is common to model the coronal magnetic field as force-free, i.e. a magnetic field where the Lorentz force is identically zero, and electric currents flow along magnetic field lines (Sturrock, 1994). Force-free models of the corona were reviewed by Sakurai (1989), and Wiegelmann and Sakurai (2012).

A force-free magnetic field satisfies (Sturrock, 1994)

$$
\nabla \times \mathbf{B}=\alpha(\mathbf{r}) \mathbf{B}
$$

and

$$
\nabla \cdot \mathbf{B}=0
$$

where the scalar field $\alpha(\mathbf{r})$ is related to the electric current density in the volume $\mathbf{J}$ by

$$
\mathbf{J}=\alpha(\mathbf{r}) \mathbf{B} / \mu_{0} .
$$

Equations (11) and (2) require boundary conditions to determine a solution, and this defines the force-free boundary value problem. The correct boundary conditions for a well-posed formulation of the problem were outlined by Grad and Rubin (1958). In the Grad-Rubin formulation the boundary conditions are the normal component of the magnetic field, $B_{n}$, and the value of $\alpha$ over one polarity of the field in the boundary, i.e. values of $\alpha$ are specified over points in the boundary where $B_{n}<$ 0 , or where $B_{n}>0$. For modeling the coronal magnetic field the boundary conditions are assumed at the photosphere and the model is solved in the coronal volume. In theoretical studies the boundary conditions usually have an analytic form (e.g. Régnier 2009, 2012), and in observationally-based studies the boundary conditions are typically derived from spectro-polarimetric observations of the photospheric mag- 
\begin{tabular}{l|l||l||l}
\hline netic field (e.g. & Schrijver et al. & 2008; De Rosa et al. & 2009, and also see references in
\end{tabular} Wiegelmann and Sakurai 2012).

The force-free boundary value problem is nonlinear in the general case where $\alpha$ is a function of position. When $\alpha$ is constant the equations are linear and closed form analytic solutions can be found (Nakagawa and Raadu, 1972; Alissandrakis, 1981). However, the linear model is unphysical in that the solutions in general have unbounded energy in an unbounded space (Alissandrakis, 1981). For the nonlinear problem, analytic solutions can be found using the generating function method (Sturrock, 1994) for particular symmetries, for example for rotational symmetry (Low and Lou, 1990). The general nonlinear problem has no known analytic solution and must be treated numerically. For this purpose a number of methods have been developed. These methods differ in their formulation of the boundary value problem and their choice of solution method (for reviews see Wiegelmann (2008) and Wiegelmann and Sakurai (2012)).

Most of the force-free methods in use solve the force-free equations in Cartesian geometry, with the corona corresponding to the half-space $z>0$ and the photosphere represented by the $z=0$ plane (Sakurai, 1981; Wu et al., 1990; Wheatland. Sturrock. and Roumeliotis, 2000; Wiegelmann, 2004; Wheatland, 2007; Valori. Kliem. and Keppens, 2005). This introduces two problems into the modeling. The first problem is that the Cartesian approximation, which assumes that the curvature of the Sun in negligible, becomes inaccurate when considering large regions on the Sun (Gary and Hagyard, 1990). Full-disk spectro-polarimetric observations of the photospheric magnetic field are now available from the Helioseismic and Magnetic Imager (HMI: Schou et al.|2012) aboard the Solar Dynamics Observatory (SDO: Pesnell. Thompson. and Chamberlin. 2012). Coronal field modeling based on these data must use spherical coordinates. The second problem concerns the assumption of boundary conditions on the transverse boundaries of the Cartesian domain. In practice the infinite half-space is replaced by a finite numerical domain, meaning boundary conditions are required on the top and side boundaries of the volume in addition to the $z=0$ plane. In general, ad hoc boundary conditions are used, such as assuming periodicity, or assuming that no magnetic flux leaves the top or side boundaries. These boundary conditions are artificial and do not necessarily represent physical conditions in the corona. Spherical modeling avoids this problem, because 
a spherical domain can encompass the entire corona with no transverse boundaries, in which case ad hoc boundary conditions are not required.

Modeling the entire corona avoids the need for boundary conditions apart from those at the photosphere, but introduces other difficulties. The description of the polar field presents difficulties both observationally and numerically. The observational difficulties are two-fold. Firstly, due to the Sun's tilt, only one pole is observed from Earth at a time. Secondly, spectro-polarimetrically derived magnetic field values may be inaccurate near the poles where there may be significant unresolved mixed-polarity magnetic flux. This can lead to partial cancellation of the polarization signal at each pixel. Quiet-Sun regions also contain mixed polarity flux, but the problem is likely to be worse close to the poles due to line-of-sight effects. For these reasons the polar field is usually interpolated from observations at lower latitudes (e.g. Sun et al. 2011). The co-ordinate singularities in the spherical polar system which occur at the poles also pose a problem for numerical methods. For finite difference methods the coordinate poles require special treatment, and with spectral methods specific grids are required to avoid problems (Boyd, 2001).

In this paper we outline an implementation of the Grad-Rubin method for solving the nonlinear force-free model in spherical geometry. Our method is applicable both to the entire Sun and to regions with restricted angular extent. Other methods for solving the force-free model in spherical geometry have been developed. Wiegelmann (2007) presented a generalization of the optimization method, and Amari et al. (2013) presented a finite-difference implementation of the Grad-Rubin method. Our method differs from both of these and features a new spectral method for computing the magnetic field based on an expansion of the field in terms of global basis functions. This solution can be applied to a spherical region of the corona of arbitrary angular size, in which case explicit assumptions about the magnetic field on the transverse boundaries of the spherical region are not required, even for a spherical wedge with restricted extent. However, for the latter case we impose additional constraints on the boundary conditions at the photosphere. Specifically, we assume that the radial component of the field and the electric current density vanish at the photosphere outside the wedge. We present the application of our method to a simple test case with analytic boundary 
conditions to demonstrate the convergence of the method and the self-consistency of the solution.

This paper is structured as follows. In Section 2 we outline the specific form of the force-free boundary value problem that we solve, and we outline the Grad-Rubin iteration method. In Section 3 we present the details of our implementation of the Grad-Rubin method, including the spectral solution for the magnetic field that is used. In Section 4 we describe the test case that we use and show the results of applying the code to the test case. Finally, in Section 5 we present a discussion of the results and a conclusion. 


\section{Theory}

In this section we outline the two boundary value problems that we solve, and give a brief description of the Grad-Rubin method. We consider solutions of the nonlinear force-free Equations (11) and (2) in two domains. The first domain is the entire corona, and the second is a spherical wedge of limited angular extent.

\subsection{Boundary value problems}

We first consider the domain $\Omega_{\text {global }}$ which is defined as the set of points with spherical polar coordinates

$$
\Omega_{\text {global }}=\left\{(r, \theta, \phi) \mid r \in\left[R_{\odot}, \infty\right), \theta \in(0, \pi), \phi \in[0,2 \pi)\right\},
$$

where $\theta$ is the polar angle, $\phi$ is the azimuthal angle, $r$ is the radius, and $R_{\odot}$ is the radius of the Sun. We refer to this domain as global because it covers a complete $4 \pi$ steradians, and its lower boundary is the entire photosphere.

It is necessary to specify boundary conditions on the force-free equations at the photosphere. Following the prescription of Grad and Rubin (1958), the appropriate boundary conditions are the radial component of $\mathbf{B}$,

$$
\left.\mathbf{B} \cdot \hat{\mathbf{r}}\right|_{r=R_{\odot}}=B_{n}(\theta, \phi),
$$

and the force-free parameter

$$
\left.\alpha\right|_{r=R_{\odot}}=\alpha_{0}(\theta, \phi)
$$

over one polarity of $B_{n}$, i.e. values of $\alpha_{0}$ are specified either where $B_{n}<0$ or where $B_{n}>0$. It is also assumed that the magnetic field vanishes for large $r$, i.e.

$$
\lim _{r \rightarrow \infty} \mathbf{B}=0 .
$$

This asymptotic boundary condition matches that used by some Cartesian codes (Wheatland, 2007). 
In some cases $B_{n}$ and $\alpha_{0}$ may only be non-zero over a small range of $\theta$ and $\phi$. In this situation it is unnecessary to use a global domain. A more appropriate choice is

$$
\Omega_{\text {wedge }}=\left\{(r, \theta, \phi) \mid r \in\left[R_{\odot}, \infty\right), \theta \in\left[\theta_{\min }, \theta_{\max }\right], \phi \in\left[\phi_{\min }, \phi_{\max }\right]\right\},
$$

i.e. a domain external to a sphere but restricted in angular extent. We refer to this as a spherical wedge. In principle, it is necessary to prescribe boundary conditions at the transverse boundaries of this domain, but in Section 3 we explain how to obtain solutions for which this is unnecessary. This approach assumes that $B_{n}$ and $\alpha_{0}$ are zero everywhere outside the domain $\Omega_{\text {wedge }}$.

Here we are using the Grad-Rubin boundary conditions which assume $\alpha_{0}$ over a single polarity of $B_{n}$, but observational data provides $\alpha_{0}$ over both (Landi Degl'Innocenti and Landolfi, 2004). This means two possible solutions can in principle be found for a given data set, corresponding to the two choices of polarity. If the data are consistent with the force-free model, then the two solutions will be the same. However, in practice it is found that the two solutions differ significantly (e.g. Schrijver et al. 2008). This may be attributed to the departure of the photospheric field from the force-free state due to significant pressure and gravity forces (Metcalf et al., 1995). Wheatland and Régnier (2009) presented a method, based on an implementation of the Grad-Rubin method in Cartesian coordinates, for using the data from both polarities to construct a single self-consistent force-free solution. Here we present only the basic Grad-Rubin method, but in principle the Wheatland and Régnier (2009) self-consistency procedure may be applied here also.

\subsection{Grad-Rubin iteration}

The Grad-Rubin method is an iterative method for solving the nonlinear force-free equations (Grad and Rubin, 1958). The method has previously been implemented in Cartesian coordinates (Sakurai, 1981; Amari. Boulmezaoud, and Mikid, 1999; Wheatland 2007) and in spherical coordinates (Amari et al., 2013). The method replaces the nonlinear Equations (11) and (2) with a pair of linear equations which are solved repeatedly in a sequence of iterations. We denote a quantity after $n$ Grad-Rubin iterations using a superscript in square brackets, e.g. $\mathbf{B}^{[n]}$. One Grad-Rubin iteration may be written 


$$
\nabla \alpha^{[n+1]} \cdot \mathbf{B}^{[n]}=0,
$$

and

$$
\nabla \times \mathbf{B}^{[n+1]}=\alpha^{[n+1]} \mathbf{B}^{[n]} .
$$

Equation (9) updates the force-free parameter $\alpha$ in the volume subject to boundary conditions on $\alpha$. Equation (10) updates the magnetic field in the volume using the new $\alpha$ values together with the magnetic field from the previous iteration subject to the boundary conditions on the normal component of the field. Equations (9) and (10) are repeatedly solved until the magnetic field $\mathbf{B}^{[n]}$ and the force-free parameter $\alpha^{[n]}$ converge at all points in the volume. The iteration is initiated using a potential field $\mathbf{B}_{0}$ constructed from the boundary conditions on $B_{n}$. We present the details of our method for solving Equations (9) and (10) numerically in two spherical domains in Section 3 . 


\section{Numerical implementation}

In this section we outline our implementation of the Grad-Rubin method in code. The Grad-Rubin method requires an initial potential field, a method for solving Equation (9) to update $\alpha$, and a method for solving Equation (10) to update the magnetic field.

The numerical grid used is a spherical polar grid with $N_{r}$ points in the $r$ direction, $N_{\theta}$ points in the $\theta$ direction, and $N_{\phi}$ points in the $\phi$ direction. The $\phi$ and $r$ grids are uniformly spaced. The $\theta$ grid is either a Gauss-Legendre grid or is uniform (Press et al., 2007). The Gauss-Legendre grid is required to accurately represent the solution near the poles and is only necessary for constructing solutions in the global domain. The grid is finite in the radial direction, and has a maximum $r$ value which we call $R_{\max }$.

\subsection{Spectral solution for the potential field}

An initial potential field is calculated as a starting point for the Grad-Rubin iteration. We use a spherical harmonic solution for the potential field. The spherical harmonics are global basis functions, meaning they are orthogonal over the domain $\Omega_{\text {global }}$. It can be shown that, in terms of spherical harmonics $Y_{l m}(\theta, \phi)$, the components of the potential field satisfying the boundary condition Equation (7) at infinity are (Altschuler and Newkirk, 1969)

$$
\begin{gathered}
B_{r}=\sum_{l=0}^{\infty} \sum_{m=-l}^{l} a_{l m}\left(\frac{R_{\odot}}{r}\right)^{(l+2)} Y_{l m}(\theta, \phi), \\
B_{\theta}=\sum_{l=0}^{\infty} \sum_{m=-l}^{l}-\frac{a_{l m}}{l+1}\left(\frac{R_{\odot}}{r}\right)^{(l+2)} \frac{\partial Y_{l m}(\theta, \phi)}{\partial \theta}
\end{gathered}
$$

and

$$
B_{\phi}=\sum_{l=0}^{\infty} \sum_{m=-l}^{l}-\frac{i m a_{l m}}{l+1}\left(\frac{R_{\odot}}{r}\right)^{(l+2)} \frac{Y_{l m}(\theta, \phi)}{\sin \theta}
$$

where the coefficients $a_{l m}$ are given by

$$
a_{l m}=\int_{0}^{2 \pi} \int_{0}^{\pi} B_{n}(\theta, \phi) Y_{l m}^{*}(\theta, \phi) \sin \theta d \theta d \phi,
$$


and where $i^{2}=-1$. These equations are complex valued and the physical magnetic field is the real part. Equations (11)-(14) can be obtained from the well-known potential source-surface solution (Altschuler and Newkirk, 1969) by considering that solution in the limit where the source surface is located at infinity.

In practice the series must be truncated after a finite number of terms. We truncate the series at a finite $l$ value which we call $L$, i.e. we perform the summation over all the spherical harmonics with $l \leq L$ and $|m| \leq l$. This approach results in a truncation error in B which is position independent (Boyd, 2001). The series is a Fourier series in $\phi$, so it is natural to choose $L$ to correspond to the Nyquist frequency (Boyd, 2001):

$$
L=\frac{\pi}{\Delta \phi}
$$

where $\Delta \phi$ is the uniform spacing of points in $\phi$. In practice the right hand side of Equation (15) is rounded to the nearest integer.

Equations (11)-(14) provide the magnetic field at all points in the global domain $\Omega_{\text {global }}$. The solution in the restricted domain $\Omega_{\text {wedge }}$ can be found by evaluating the global solution only at points contained in $\Omega_{\text {wedge. }}$. This allows the solution in $\Omega_{\text {wedge }}$ to be found without assuming specific boundary conditions on the transverse boundaries of $\Omega_{\text {wedge }}$. Since we are assume that $B_{n}$ is zero outside $\Omega_{\text {wedge }}$, the integral in Equation (14) need only be computed over the restricted domain.

Tóth, van der Holst, and Huang (2011) report the non-convergence of the spectral series given by Equations (12)-(13). The non-convergence results in erroneous magnetic field values (particularly near the poles) for large $L$. The problem occurs because the numerical grid used by Tóth. van der Holst, and Huang (2011) is not sufficiently dense near the poles to accurately represent the rapid variation of the spherical harmonics. For calculations in $\Omega_{\text {global }}$ we use a Gauss-Legendre grid which accurately represents the spherical harmonics near the poles (Bovd, 2001). For calculations in $\Omega_{\text {wedge, }}$, we use uniform grid in $\theta$, which is rotated such that the region of interest is isolated from the poles, and does not encounter this problem.

The Gibbs phenomenon (ringing produced in representing discontinuous changes) is a problem for all spectral methods (Bovd, 2001). The problem is significant when spectral potential field solutions are calculated from observational data (Tóth. van der Holst, and Huang, 
2011). Including more terms in the series improves but does not eliminate the problem.

It is important to note this particular caveat when applying and interpreting results produced by spectral methods. It should be noted that finite difference methods also become inaccurate at locations with large gradients in the field being represented.

A parallel code is used to sum the spectral series. The coefficients $a_{l m}$ are calculated using Equation (14) and then Equations (11)-(13) are evaluated with the sums performed using partial sums, i.e. each series is broken into a number of sub-series, each of which is summed independently, and then the final result is obtained by adding the partial sums. The parallel implementation is written for a distributed memory multiprocessor. The method uses a combination of the Message Passing Interface (MPI) (Snir et al., 1998) and OpenMP (Chandra et al., 2001) and is described in Appendix B.

3.2. Field line tracing solution for the current-update step

To solve Equation (9) we employ the field line tracing method which has been used in Grad-Rubin implementations in Cartesian coordinates (Amari. Boulmezaoud, and Mikid, 1999; Wheatland, 2007), and in spherical coordinates (Amari et al., 2013). According to Equation (9), $\alpha^{[n+1]}$ is constant along magnetic field lines. The field line tracing method determines $\alpha^{[n+1]}$ in the volume by tracing the field line threading each grid point until it crosses the lower boundary, and the value of $\alpha_{0}$ at the crossing point in the boundary is assigned to the grid point. The field line is traced in the forwards direction if boundary values for $\alpha_{0}$ are chosen where $B_{n}<0$, and is traced in the backwards direction if boundary values for $\alpha_{0}$ are chosen where $B_{n}>0$. Points in the volume connected to field lines which leave the domain through the outer boundary $r=R_{\max }$ are assigned $\alpha^{[n+1]}=0$ at the point in the volume. In addition, points in the volume threaded by field lines which leave the transverse boundaries of $\Omega_{\text {wedge }}$ are assigned $\alpha^{[n+1]}=0$ at the point in the volume. The tracing is performed using fourthorder Runge-Kutta integration (Press et al., 2007), and trilinear interpolation is used to determine $\mathbf{B}^{[n]}$ at points along the field line not coinciding with a grid point. 
3.3. Spectral solution to Ampere's law for the field-update step

To solve Equation (10) we use a spectral solution. The magnetic field is decomposed into the sum of a potential field and a non-potential field, i.e.

$$
\mathbf{B}^{[n+1]}=\mathbf{B}_{0}+\mathbf{B}_{c}^{[n+1]}
$$

where $\mathbf{B}_{c}^{[n+1]}$ satisfies

$$
\nabla \times \mathbf{B}_{c}^{[n+1]}=\mathbf{J}^{[n+1]},
$$

with

$$
\mathbf{J}^{[n+1]}=\alpha^{[n+1]} \mathbf{B}^{[n]} / \mu_{0},
$$

and where $\mathbf{B}_{0}$ is the potential field matching the boundary conditions on $B_{n}$ calculated using the method of Section 3.1 It is only necessary to update $\mathbf{B}_{c}^{[n+1]}$ at each iteration as $\mathbf{B}_{0}$ does not change. Also, since $\mathbf{B}_{0}$ satisfies the boundary conditions on the normal component of the field at $r=R_{\odot}$ it follows that

$$
\left.\mathbf{B}_{c}^{[n+1]}\right|_{r=R_{\odot}}=0,
$$

and from Equation (77) we require

$$
\lim _{r \rightarrow \infty} \mathbf{B}_{c}^{[n+1]}=0
$$

Equations (19) and (20) define the boundary conditions on $\mathbf{B}_{c}^{[n+1]}$.

We use a spectral solution to Equation (17) which is analogous to the spherical harmonic solution for the potential field. We express $\mathbf{B}_{c}^{[n+1]}$ as a series using the vector spherical harmonics (Morse and Feshbach, 1953), i.e.

$$
\mathbf{B}_{c}^{[n+1]}=\sum_{l=0}^{\infty} \sum_{m=-l}^{l} B_{l m}^{(1)}(r) \mathbf{Y}_{l m}+B_{l m}^{(2)}(r) \mathbf{\Psi}_{l m}+B_{l m}^{(3)}(r) \mathbf{\Phi}_{l m}
$$


where $\mathbf{Y}_{l m}, \mathbf{\Psi}_{l m}$, and $\boldsymbol{\Phi}_{l m}$ are the complete set of orthogonal vector basis functions defined by

$$
\begin{gathered}
\mathbf{Y}_{l m}=Y_{l m} \hat{\mathbf{r}} \\
\mathbf{\Psi}_{l m}=\frac{r \nabla Y_{l m}}{\sqrt{l(l+1)}},
\end{gathered}
$$

and

$$
\mathbf{\Phi}_{l m}=\frac{\mathbf{r} \times \nabla Y_{l m}}{\sqrt{l(l+1)}} .
$$

These functions are mutually perpendicular, i.e.

$$
\mathbf{Y}_{l m} \cdot \mathbf{\Psi}_{l m}=\mathbf{Y}_{l m} \cdot \boldsymbol{\Phi}_{l m}=\mathbf{\Psi}_{l m} \cdot \mathbf{\Phi}_{l m}=0
$$

and orthonormal, e.g.

$$
\int_{0}^{\pi} \int_{0}^{2 \pi} \mathbf{Y}_{l m}(\theta, \phi) \cdot \mathbf{Y}_{l^{\prime} m^{\prime}}^{*}(\theta, \phi) d \theta d \phi=\delta_{l l^{\prime}} \delta_{m m^{\prime}}
$$

where $\delta_{l m}$ is the Kronecker delta1 1 . The vector spherical harmonics have previously been applied to magnetostatic problems (e.g. Barrera, Estévez, and Giraldo, 1985; Dennis and Quartapelle 1985), but have not been used in this context.

The spectral coefficients $B_{l m}^{(i)}$ with $i=1,2,3$ are determined by the distribution of currents in the volume and by the boundary conditions. We show in Appendix A that for the problem at hand the spectral coefficients for the magnetic field are

$$
\begin{gathered}
B_{l m}^{(1)}=\frac{\sqrt{l(l+1)}}{r}\left[-R_{\odot}^{l}\left(\frac{R_{0}}{r}\right)^{l+1} I_{0}+I_{2}(r)+I_{3}(r)\right], \\
B_{l m}^{(2)}=\frac{1}{r}\left[R_{\odot}^{l}\left(\frac{R_{\odot}}{r}\right)^{l+1} I_{0}-l I_{1}(r)+(l+1) I_{2}(r)\right],
\end{gathered}
$$

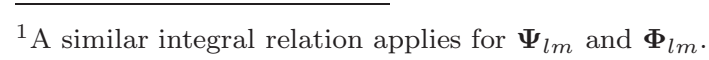


and

$$
B_{l m}^{(3)}=\frac{r J_{l m}^{(1)}}{\sqrt{l(l+1)}}
$$

where

$$
\begin{gathered}
I_{0}=\frac{\mu_{0}}{2 l+1} \int_{R_{\odot}}^{\infty} s^{1-l} J_{l m}^{(3)}(s) d s, \\
I_{1}(r)=\frac{\mu_{0}}{2 l+1} \int_{R_{\odot}}^{r} s\left(\frac{s}{r}\right)^{l+1} J_{l m}^{(3)}(s) d s,
\end{gathered}
$$

and

$$
I_{2}(r)=\frac{\mu_{0}}{2 l+1} \int_{r}^{\infty} s\left(\frac{r}{s}\right)^{l} J_{l m}^{(3)}(s) d s .
$$

The coefficients $J_{l m}^{(i)}$ are the spectral coefficients of the current distribution defined by

$$
\begin{aligned}
& J_{l m}^{(1)}(r)=\int \mathbf{J}^{[n+1]} \cdot \mathbf{Y}_{l m}^{*} d \Omega, \\
& J_{l m}^{(2)}(r)=\int \mathbf{J}^{[n+1]} \cdot \mathbf{\Psi}_{l m}^{*} d \Omega,
\end{aligned}
$$

and

$$
J_{l m}^{(3)}(r)=\int \mathbf{J}^{[n+1]} \cdot \boldsymbol{\Phi}_{l m}^{*} d \Omega,
$$

where $\mathbf{J}^{[n+1]}$ is the volume current density defined by Equation (18). The spectral solution is computed in three steps: i) $J_{l m}^{(i)}$ is computed from $\mathbf{J}^{[n+1]}$ using Equations (33)-(35); ii) spectral coefficients for the magnetic field are computed from Equations (27)-(29); iii) the spectral series is summed to a maximum order $L$. A parallel summation method is used, as described in Appendix B (the method is described for the potential field calculation, but the same approach is also used for the non-potential component of the field).

The integrals $I_{0}, I_{1}$, and $I_{2}$ [Equations (30), (31), and (32)] are evaluated using the trapezoidal rule. The integrals are expressed in a such a way as to avoid numerical overflow for large values of $l$. Although the integrals in Equations (30) and (32) are written as extending to infinite radius, in the numerical solution the maximum radius 
is $R_{\max }$, and an error is introduced by this approximation if $\mathbf{J}^{[n+1]} \neq 0$ at $r=R_{\max }$. Therefore it is necessary to make $R_{\max }$ sufficiently large to encompass all the significant currents.

SOLA: sphere_nlfff_paper.tex; 11 June 2021; 15:22; p. 15 


\section{Application to test cases}

In this section we apply our code to two test cases with analytic boundary conditions, to demonstrate the method. We establish the convergence of the method and quantify the self-consistency of the solution.

\subsection{Two bipolar test cases}

The first test case is a simple model with analytic boundary conditions, representing the field due to a bipolar active region with Gaussian sunspots calculated in the global domain $\Omega_{\text {global }}$. A small non-zero patch of $\alpha$ is included around one of the spots. The second test case has the same boundary conditions, on a smaller spacial scale, and the field is calculated in a restricted domain $\Omega_{\text {wedge }}$. For each test case we demonstrate the convergence of the Grad-Rubin iteration, and we measure the self-consistency of the solution by verifying that the Lorentz force in the model corona is zero, i.e. that the electric current is parallel to the magnetic field.

For both test cases, the boundary conditions on the magnetic field are

$$
B_{n}(\theta, \phi)=B_{\mathrm{s}}\left(e^{-s_{1}^{2} / \sigma^{2}}-e^{-s_{2}^{2} / \sigma^{2}}\right)
$$

where $B_{\mathrm{s}}$ is a scale constant which is chosen such that $\max \left(\left|B_{n}\right|\right)=B_{\mathrm{s}}$, and $\sigma$ is a parameter which determines the size of the spots. The two functions $s_{1}$ and $s_{2}$ are distances to the centers of each spot as measured on the sphere, and may be written

$$
s_{i}(\theta, \phi)=R_{\odot} \tan ^{-1}\left[\frac{\sqrt{(\sin \theta \sin \Delta \lambda)^{2}+\left(\sin \theta_{i} \cos \theta-\cos \theta \sin \theta_{i} \cos \Delta \lambda\right)^{2}}}{\cos \theta_{i} \cos \theta+\sin \theta \sin \theta_{i} \cos \Delta \lambda}\right],
$$

where $\Delta \lambda=\phi_{i}-\phi$, and with $\left(\theta_{1}, \phi_{1}\right)$ being the coordinates of the center of the spot with positive polarity, and with $\left(\theta_{2}, \phi_{2}\right)$ being the coordinates of the center of the spot with negative polarity. For the boundary conditions on $\alpha_{0}$ we consider an isolated spot with a locally constant value of $\alpha$ around the center of the positive spot, i.e.

$$
\alpha_{0}=\left\{\begin{array}{ll}
\Lambda & B_{n} \geq B_{\mathrm{th}} \\
0 & 0<B_{n}<B_{\mathrm{th}}
\end{array},\right.
$$


where $\Lambda$ is a constant, and $B_{\text {th }}$ is a threshold value. Note that the boundary conditions on $\alpha_{0}$ are only defined over one polarity of $B_{n}$ (as per the Grad-Rubin formulation). We have chosen to define $\alpha_{0}$ over the positive polarity of $B_{n}$, i.e. where $B_{n}>0$.

It is known that force-free fields can be unstable when

$$
\alpha_{\mathrm{s}} L_{\mathrm{s}}>1
$$

(Molodensky, 1974), where $\alpha_{\mathrm{s}}$ is a characteristic value for $\alpha$, and $L_{\mathrm{s}}$ is the characteristic scale length. For the bipolar test case we take the scale length to be the distance along the photosphere between the two spots, and we take $\alpha_{\mathrm{s}}=\Lambda$. For both test cases we choose these values such that

$$
\alpha_{\mathrm{s}} L_{\mathrm{s}}=1
$$

corresponding to a current matching the approximate maximum.

4.2. Measures of convergence

To measure the convergence of the iteration we employ two metrics. The first is the total magnetic energy at each iteration $n$ :

$$
E_{n}=\frac{1}{2 \mu_{0}} \int\left|\mathbf{B}^{[n]}\right|^{2} d V
$$

where the integral is over the entire computational volume. We expect the energy to converge to a fixed value with Grad-Rubin iteration. The second metric is the average absolute difference in the magnetic field at successive Grad-Rubin iterations:

$$
A_{\text {mean }}=\left\langle\left|\mathbf{B}^{[n]}-\mathbf{B}^{[n-1]}\right|\right\rangle
$$

where $\langle\ldots\rangle$ denotes the average over the computational volume. We expect $A_{\text {mean }}$ to approach zero with repeated iteration. 
To measure the self consistency of the solution, we consider the maximum angle between $\mathbf{J}$ and $\mathbf{B}$. The angle is defined by

$$
\theta_{\max }=\max \left[\frac{\left|\mathbf{J}^{[n]} \times \mathbf{B}^{[n]}\right|}{\left|\mathbf{B}^{[n]}\right|\left|\mathbf{J}^{[n]}\right|}\right]
$$

where the maximum is calculated over all grid points.

We also examine the self-consistency qualitatively by drawing the field lines of $\mathbf{B}^{[n]}$ and streamlines of the current density $\mathbf{J}^{[n]}$. For an exact force-free solution we expect that these two sets of field lines will coincide corresponding to $\theta_{\max }=0$. This provides quite a stringent test, because the error in the paths of the field lines is the result of the local truncation error in the solution integrated along the paths, which is in general greater than the local error.

\subsection{Test case one: the bipole in the global domain}

The first test case described in Section 4.1 considers a bipolar active region covering a significant fraction of the photosphere. This region provides a test for the method in the domain $\Omega_{\text {global }}$. The parameters used for this test are shown in Table 1 and the dimensions of the grid are summarized in Table2, We perform 40 Grad-Rubin iterations starting from a potential field. The computation takes approximately 15 minutes on a computer with an eight-core CPU.

The left panel of Figure@illustrates the energy (in units of the energy of the potential field $\left.E_{0}\right)$ as a function of iteration number. The energy converges to an approximately constant value after about six Grad-Rubin iterations. The left panel of Figure 2 shows the absolute average change in the field, $A_{\text {mean }}$, as a function of iteration number. The scale on the $y$ axis is logarithmic. This figure shows that $A_{\text {mean }}$ decreases exponentially before becoming roughly constant. The behavior of the two metrics $E / E_{0}$ and $A_{\text {mean }}$ establishes the convergence of the Grad-Rubin procedure for this case.

We also demonstrate the self-consistency of the numerical solution. Figure 3 shows the field lines of $\mathbf{B}$ (in blue) and the streamlines of $\mathbf{J}$ (in red). The left panel shows the results after one Grad-Rubin iteration. In this panel there is a significant discrepancy between the two sets of lines. The right panel shows the field lines after 40 GradRubin iterations. In this case the two sets of lines almost coincide, indicating that the 
numerical solution is close to being force-free. Some discrepancy is observed for long field lines because of the need to interpolate values of $\mathbf{J}$ and $\mathbf{B}$ between grid points in order to draw field lines. At each grid point $\mathbf{J} \times \mathbf{B}$ is very small (as discussed below), but larger values are obtained between grid points when $\mathbf{J}$ and $\mathbf{B}$ are interpolated. The field lines which show the largest discrepancy occur close to the boundary separating zero and non-zero values of $\alpha_{0}$, where the interpolation is least accurate.

The left panel of Figure 4 shows $\theta_{\max }$ as a function of iteration number. After the first iteration the maximum angle between $\mathbf{J}$ and $\mathbf{B}$ is approximately 20 degrees, and $\theta_{\max }$ decreases approximately exponentially over the 40 iterations to a final value of order $10^{-3}$ degrees. This confirms that a force-free solution is found.

\subsection{Results for test case two: the bipole in the restricted domain}

The second test case described in Section 4.1 considers the bipolar test case in the domain $\Omega_{\text {wedge }}$. We choose a domain which spans $20^{\circ}$ in latitude and $20^{\circ}$ in longitude. The size and the dimensions of the numerical grid are summarized in Table 2, In this case, the separation between the spots is an order of magnitude smaller than for the first test case, so we increase $\alpha_{\mathrm{s}}$ by an order of magnitude so that the product $L_{\mathrm{s}} \alpha_{\mathrm{s}}$ matches test case one. This provides a bipole with the same amount of twist. The parameters for this test case are summarized in Table2, We again apply 40 Grad-Rubin iterations, which takes 1.6 hours to run on a computer with an eight-core CPU.

The right panel of Figure 1 shows the energy (in units of the energy of the potential field $\left.E_{0}\right)$ as a function of iteration number. The energy converges to a constant value after approximately four Grad-Rubin iterations. The right panel of Figure 2 shows $A_{\text {mean }}$ as a function of iteration number. The scale on the $y$ axis is logarithmic, and the figure shows that $A_{\text {mean }}$ decreases approximately exponentially, to a final value the order of machine precision. The behavior of $E / E_{0}$ and $A_{\text {mean }}$ as a function of iteration confirm the convergence of the Grad-Rubin iteration procedure.

The left panel of Figure 5 shows the field lines of the magnetic field and the streamlines of the current density after the first iteration (left panel) and after the last iteration (right panel). The magnetic field is shown in blue and the current density in red. In the left panel there is a clear difference between the two sets of lines, and in the right 
panel the two sets of lines closely coincide, indicating that a force-free solution is found. Some disagreement is observed between the two sets of lines for longer field lines and is explained in Section 4.3

The right panel of Figure 4 shows $\theta_{\max }$ as a function of iteration number. After the first iteration the maximum angle between $\mathbf{J}$ and $\mathbf{B}$ is approximately 20 degrees, and $\theta_{\max }$ decreases approximately exponentially with iteration, to a final value of order $10^{-9}$ degrees.

\section{Discussion and conclusion}

We present an implementation of the Grad-Rubin method (Grad and Rubin, 1958) for solving the force-free boundary value problem in spherical polar geometry with the aim of modeling the solar coronal magnetic field. The method is applicable to either the entire corona or a wedge with restricted angular extent.

We apply our code to two test cases with analytic boundary conditions, representing a current-carrying bipole, to demonstrate the convergence of the Grad-Rubin iteration and the self-consistency of the final numerical solution. For the first case we compute a solution in a domain which encompasses the entire corona, and in the second we compute a solution in a spherical wedge. We find that in both cases the Grad-Rubin iteration converges, and the resulting solution is force free to a very good approximation, by which we mean that the angle between $\mathbf{J}$ and $\mathbf{B}$ is small.

The bipolar test case we consider is simple, but serves to illustrate the basic correctness of the method. We are unable to identify a suitable exact analytic equilibria with which to test the code. The Low and Lou (1990) field has often been used for testing force-free codes (Amari. Boulmezaoud, and Mikic, 1999; Wiegelmann, 2007), but it proves difficult to reproduce this solution without imposing analytic boundary conditions on an outer shell located at a finite radius from the photosphere. Using the asymptotic boundary condition defined by Equation (7), requires a very large domain to encompass all the significant currents for the Low and Lou (1990) problem. Hence it is difficult to calculate accurate solutions without resorting to the use of exact boundary conditions at a finite outer shell. The lack of an exact analytic test case means that we cannot determine how the numerical error scales as a function of grid resolution. We 
also note that our test case has smooth boundary conditions. In practice, we expect observational data to contain significant gradients, in particular due to noise which is present in the data. The effect of Gibbs phenomenon produced by the spectral method applied to these large gradients has not yet been investigated.

We note that the code takes 1.6 hours to run for the test case in the spherical wedge, for a grid with $64^{3}$ points. By comparison the calculation in the global domain takes 15 minutes for a $128 \times 64 \times 128$ grid. The difference in speed occurs because the spherical harmonics must be summed to large orders when $\Delta \phi$ is small. In the first case $L=64$ and in the second case $L=567$. In practice it may not be necessary to use an $L$ value of a given size for all values of $r$. We expect that as the field becomes smoother with increasing $r$ higher order terms may be ignored and a reduction in $L$ as a function of $r$ may increase the speed of the code. Also, we expect that it may be possible to increase the speed of the code by improving details of the technique used for the calculation of the non-potential field. These improvements will be implemented in future work.

As mentioned in Section 3.1 Tóth. van der Holst, and Huang (2011) report the nonconvergence of the spherical harmonics spectral series for large $L$, for the calculation of potential field models. The grid used by Tóth. van der Holst, and Huang (2011) is uniform in $\cos \theta$. However, near the poles, the associated Legendre polynomials are rapidly varying functions of $\cos \theta$, and the rapid variations are not accurately represented on the given grid. This results in the non-convergence of the series. For our calculations in $\Omega_{\text {wedge }}$, we use a grid uniform in $\theta$. Tóth, van der Holst, and Huang (2011) report that such a grid does not cause convergence problems. Furthermore, the region considered in Section 4.4 is sufficiently isolated from the poles that convergence problems are unlikely to occur regardless of the grid used. When considering larger regions which cannot be isolated from the poles, or for calculations in $\Omega_{\text {global }}$ we use a Gauss-Legendre grid which is dense at the poles and accurately represents the rapid variations in the associated Legendre polynomials.

Other developments to the code are also planned. At present, the code uses a uniform grid in $r$, but this could be changed to a nonuniform grid. A nonuniform grid should be more efficient because the grid can be chosen to be dense close to the photosphere where the magnetic field is structured on small scales, and sparse far from the photosphere 
where the field is smooth. At present the method assumes the asymptotic boundary condition Equation (7) but this could be changed to accommodate boundary conditions at an outer "source surface" analogous to the potential source-surface model. Finally, the method presently only uses $\alpha_{0}$ over a single polarity, which is likely to be problematic when using observational data because of the inconsistency of the data with the force-free model (Schrijver et al., 2008). In future work we will modify our method to implement the self-consistency procedure of Wheatland and Régnier (2009).

The method and code outlined in this paper are designed for application to solar data, but we have not yet attempted this. In future work we will test the code on vector magnetogram data derived from observations by the Helioseismic and Magnetic Imager aboard the Solar Dynamics Observatory (SDO/HMI). The SDO/HMI instrument provides data for the whole solar disk, which demands spherical modeling. We hope to be able to develop the code presented here into a practical tool for modeling of the coronal magnetic field from SDO/HMI data, and for application to other, future data sets. 


\section{Appendix A}

In this appendix we derive Equations (27)-(29) using vector spherical harmonics.

For any magnetic field it is possible to introduce a vector potential, A, related to the magnetic field by

$$
\nabla \times \mathbf{A}=\mathbf{B} .
$$

In the Coulomb gauge,

$$
\nabla \cdot \mathbf{A}=0
$$

in which case the vector potential is given by the vector Poisson equation, (Jackson, 1998):

$$
\nabla^{2} \mathbf{A}=-\mu_{0} \mathbf{J}
$$

The boundary conditions on $\mathbf{A}$ enforcing the boundary conditions on $\mathbf{B}$ given by Equations (19) and (20) are

$$
\mathbf{A} \times \hat{\mathbf{r}}=0,
$$

and

$$
\lim _{r \rightarrow \infty} \mathbf{A}=0
$$

respectively.

We can solve Poisson's equation by expanding $\mathbf{A}$ in terms of a set of orthonormal basis functions, which reduces the partial differential equation to a system of ordinary differential equations for the series coefficients. The vector spherical harmonics provide a natural set of basis functions in spherical polar coordinates (Morse and Feshbach, 1953). The vector potential can be written in terms of these functions as:

$$
\mathbf{A}=\sum_{l=0}^{\infty} \sum_{m=-l}^{l} A_{l m}^{(1)} \mathbf{Y}_{l m}+A_{l m}^{(2)} \mathbf{\Psi}_{l m}+A_{l m}^{(3)} \boldsymbol{\Phi}_{l m},
$$

where $A_{l m}^{(i)}$ are the spectral coefficients with $i=1,2,3$. By substituting Equation (49) into Equation (46) and using the orthonormality of the basis functions, it can be shown 
that $A_{l m}^{(3)}$ satisfies the second-order linear inhomogeneous equation

$$
\frac{d^{2} A_{l m}^{(3)}}{d r^{2}}+\frac{2}{r} \frac{d A_{l m}^{(3)}}{d r}-\frac{l(l+1)}{r^{2}} A_{l m}^{(3)}=-\mu_{0} J_{l m}^{(3)}
$$

where

$$
J_{l m}^{(3)}=\int_{0}^{\pi} \int_{0}^{2 \pi} \mathbf{J} \cdot \mathbf{\Phi}_{l m} \sin \theta d \phi d \theta .
$$

Equation (50) has a general solution related to the boundary conditions on $\mathbf{A}$, and a particular solution, determined by the source terms $J_{l m}^{(3)}$. The solutions can be found analytically using the method of variation of parameters (Zwillinger, 1989). This gives

$$
A_{l m}^{(3)}(r)=-R_{\odot}^{l}\left(\frac{R_{\odot}}{r}\right)^{l+1} I_{0}+I_{1}(r)+I_{2}(r)
$$

where

$$
\begin{gathered}
I_{0}=\frac{\mu_{0}}{2 l+1} \int_{R_{\odot}}^{\infty} s^{1-l} J_{l m}^{(3)}(s) d s, \\
I_{1}(r)=\frac{\mu_{0}}{2 l+1} \int_{R_{\odot}}^{r} s\left(\frac{s}{r}\right)^{l+1} J_{l m}^{(3)}(s) d s,
\end{gathered}
$$

and

$$
I_{2}(r)=\frac{\mu_{0}}{2 l+1} \int_{r}^{\infty} s\left(\frac{r}{s}\right)^{l} J_{l m}^{(3)}(s) d s .
$$

The spectral coefficients $B_{l m}^{(1)}$ and $B_{l m}^{(2)}$ can be determined from Equation (52) as follows. Taking the curl of the vector spherical harmonics leads to the following identities

$$
\begin{gathered}
\nabla \times\left[F_{l m}(r) \mathbf{Y}_{l m}\right]=\sqrt{l(l+1)} \frac{F_{l m}(r)}{r} \boldsymbol{\Phi}_{l m}, \\
\nabla \times\left[F_{l m}(r) \mathbf{\Psi}_{l m}\right]=-\left(\frac{d}{d r}+\frac{1}{r}\right) F_{l m}(r) \mathbf{\Phi}_{l m},
\end{gathered}
$$

and

$$
\nabla \times\left[F_{l m}(r) \mathbf{\Phi}_{l m}\right]=\sqrt{l(l+1)} \frac{F_{l m}(r)}{r} \mathbf{Y}_{l m}+\left(\frac{d}{d r}+\frac{1}{r}\right) F_{l m}(r) \mathbf{\Psi}_{l m}
$$


where $F_{l m}(r)$ is a any function of $r$. Since $\mathbf{B}=\nabla \times \mathbf{A}$, it follows that

$$
\begin{aligned}
\mathbf{B} & =\sum_{l=0}^{\infty} \sum_{m=-l}^{l} \sqrt{l(l+1)} \frac{A_{l m}^{(3)}(r)}{r} \mathbf{Y}_{l m}+\left(\frac{d}{d r}+\frac{1}{r}\right) A_{l m}^{(3)}(r) \mathbf{\Psi}_{l m} \\
& +\left[\sqrt{l(l+1)} \frac{A_{l m}^{(1)}(r)}{r}-\left(\frac{d}{d r}+\frac{1}{r}\right) A_{l m}^{(2)}(r)\right] \mathbf{\Phi}_{l m} .
\end{aligned}
$$

The spectral coefficients are then given by

$$
B_{l m}^{(1)}=\sqrt{l(l+1)} \frac{A_{l m}^{(3)}(r)}{r}
$$

and

$$
B_{l m}^{(2)}=\left(\frac{d}{d r}+\frac{1}{r}\right) A_{l m}^{(3)}(r)
$$

where we use the fact that the vector spherical harmonics are orthonormal. By substituting Equation (52) into Equations (60) and (61) we find that

$$
B_{l m}^{(1)}=\frac{\sqrt{l(l+1)}}{r}\left[-R_{\odot}^{l}\left(\frac{R_{0}}{r}\right)^{l+1} I_{0}+I_{2}(r)+I_{3}(r)\right] \text {, }
$$

and

$$
B_{l m}^{(2)}=\frac{1}{r}\left[R_{\odot}^{l}\left(\frac{R_{\odot}}{r}\right)^{l+1} I_{0}-l I_{1}(r)+(l+1) I_{2}(r)\right] .
$$

It is possible to determine $B_{l m}^{(3)}$ by finding $A_{l m}^{(1)}$ and $A_{l m}^{(2)}$ in the same manner as for $A_{l m}^{(3)}$, and evaluating the expression in Equation (60) for the coefficients of $\boldsymbol{\Phi}_{l m}$. However, a simpler approach is to apply Equation (58) to Ampere's law $\nabla \times \mathbf{B}=\mu_{0} \mathbf{J}$. This yields

$$
B_{l m}^{(3)}=\frac{r J_{l m}^{(1)}}{\sqrt{l(l+1)}}
$$

where

$$
J_{l m}^{(1)}=\int_{0}^{\pi} \int_{0}^{2 \pi} \mathbf{J} \cdot \mathbf{Y}_{l m} \sin \theta d \phi d \theta
$$




\section{Appendix B}

In this appendix we outline a parallel method for evaluating the sums in Equations (11)-

(13). The method of summing the spectral series is important because it determines the speed of the computation. Also, a high resolution grid requires a large order $L$, in which case the memory required to compute the spectral solution becomes large (depending of the implementation). In the following we present a method for summing the series which is fast and which uses memory efficiently. The procedure is described for the $B_{r}$ component of the potential field. A similar approach is used for the other components of the potential field, and for the non-potential field.

5.1. Parallel summation of the spectral series

The following procedure for computing $B_{r}$ follows a prescription in Press et al. (2007). Here we briefly outline the method and describe a parallel implementation.

The spectral series for $B_{r}$ can be written as

$$
B_{r}=\sum_{m=-L}^{L} g_{m}(r, \theta) e^{i m \phi},
$$

where the auxiliary function $g_{m}(r, \theta)$ is defined as

$$
g_{m}(r, \theta)=\sum_{l=|m|}^{L} a_{l m}\left(\frac{R_{\odot}}{r}\right)^{l+2} \tilde{P}_{l}^{m}(\cos \theta)
$$

and where the spherical harmonics $Y_{l m}$ are written as a product of a normalizedassociated Legendre polynomial $\tilde{P}_{l}^{m}(x)$ and the complex exponential. In our method we first compute the auxiliary function using Equation (67), and then determine $B_{r}$ using Equation (66).

This procedure can be implemented in parallel by partitioning the sum in Equation (66) into $N$ partial sums which are computed independently. We define the partial sum

$$
F_{n}=\sum_{m=-m_{n}}^{m_{n}} g_{m}(r, \theta) e^{i m \phi}
$$


where

$$
m_{n}=n L / N
$$

The complete solution is then

$$
B_{r}=\sum_{n=0}^{L} F_{n} .
$$

Each $F_{n}$ can be computed independently, allowing trivial parallelism. Most high performance computer clusters consist of a series of nodes, with each node containing several processors with shared a memory space. Our code distributes the computation of $F_{n}$ among such nodes using the Message Passing Interface (MPI) (Snir et al., 1998). The computation of $F_{n}$ at each node is parallelized across the processors on the node using OpenMP (Chandra et al.), 2001).

\subsection{Memory requirements}

It is important to consider the memory required to store $g_{m}(r, \theta)$ as an array. Since the integrand in Equation (14) is real, the coefficients with $m<0$ contain the same information as those with $m>0$ (Press et al., 2007). Therefore, it is necessary only to store coefficients with $m \geq 0$, and there are $(L+1) L / 2$ such coefficients. Therefore an array storing $g_{m}(r, \theta)$ has $L(L+1) N_{r} N_{\theta} / 2$ elements for a grid of size $N_{r} \times N_{\theta} \times N_{\phi}$. The associated memory use may prove problematic for $L \sim 1000$. A grid of size $N_{r}=128$, $N_{\theta}=256$, and $N_{\phi}=512$ requires $\approx 100 \mathrm{Mb}$ to store $B_{r}$ using double precision floating point numbers, and $\approx 1 \mathrm{~Gb}$ to store $g_{m}(r, \theta)$. The memory requirements for $g_{m}(r, \theta)$ can quickly become larger than a few gigabytes, a typical size for total memory on current desktop computer hardware.

The partial summation method reduces the memory requirements because the array $g_{m}$ is split among the nodes. If the series is split into $N$ partial sums, then the memory requirement for each node is $\sim 1 / N$ of that needed to store the entire array. The partial summation method can also be used to conserve memory with only a single node. In this case each partial sum is computed sequentially. This way only part of the spectrum $g_{m}$ is computed for each partial summation. Again, this method reduces the memory requirements by a factor $\sim 1 / N$ compared with a single serial summation. 
Table 1. Parameters used for the bipolar test cases involving the domain $\Omega_{\text {global }}$ (case 1 ), and the wedge-shaped region $\Omega_{\text {wedge }}$ (case 2). Distances are quoted in units of a solar radius, and magnetic field strengths are relative to the scale constant $B_{\mathrm{S}}$. Units are given in square brackets.

\begin{tabular}{llllll}
\hline Case & $\begin{array}{l}\sigma \\
{\left[R_{\odot}\right]}\end{array}$ & $\begin{array}{l}L_{\mathrm{s}} \\
{\left[R_{\odot}\right]}\end{array}$ & $\begin{array}{l}\Lambda \\
{\left[R_{\odot}^{-1}\right]}\end{array}$ & $\alpha_{\mathrm{s}} L_{\mathrm{s}}$ & $\begin{array}{l}B_{\text {th }} \\
{\left[B_{s}\right]}\end{array}$ \\
\hline 1 & 0.1 & 0.35 & 3 & 1.05 & 0.75 \\
2 & 0.02 & 0.035 & 30 & 1.05 & 0.75
\end{tabular}

Table 2. Size of the grids used for test cases. Both the physical sizes and the sizes of the numerical grids are shown.

\begin{tabular}{llllllll}
\hline Case & Domain & $N_{r}$ & $N_{\theta}$ & $N_{\phi}$ & $\begin{array}{l}\text { Lon } \\
{[\text { degrees }]}\end{array}$ & $\begin{array}{l}\text { Lat } \\
{[\text { degrees }]}\end{array}$ & $\begin{array}{l}R_{\max } \\
{\left[R_{\odot}\right]}\end{array}$ \\
\hline 1 & $\Omega_{\text {global }}$ & 128 & 64 & 128 & 360 & 180 & 6 \\
2 & $\Omega_{\text {wedge }}$ & 64 & 64 & 64 & 20 & 20 & 1.2
\end{tabular}



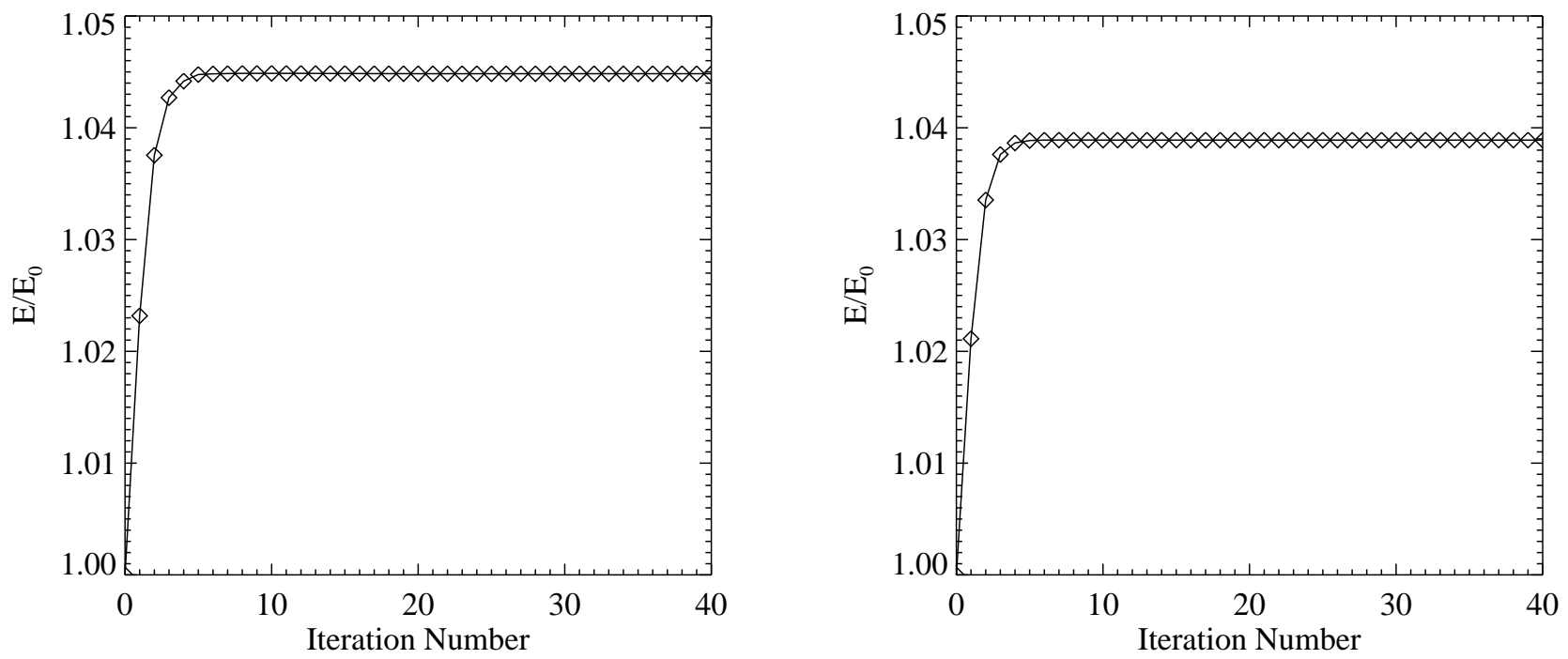

Figure 1. Energy (in units of the energy of the potential field $E_{0}$ ) as a function of iteration number. The left panel shows $E / E_{0}$ for the first test case in the global domain $\Omega_{\text {global }}$, and the right panel shows the same for the second test case, involving a calculation in the domain $\Omega_{\text {wedge }}$. In both cases the energy converges to an approximately constant value after about six iterations.
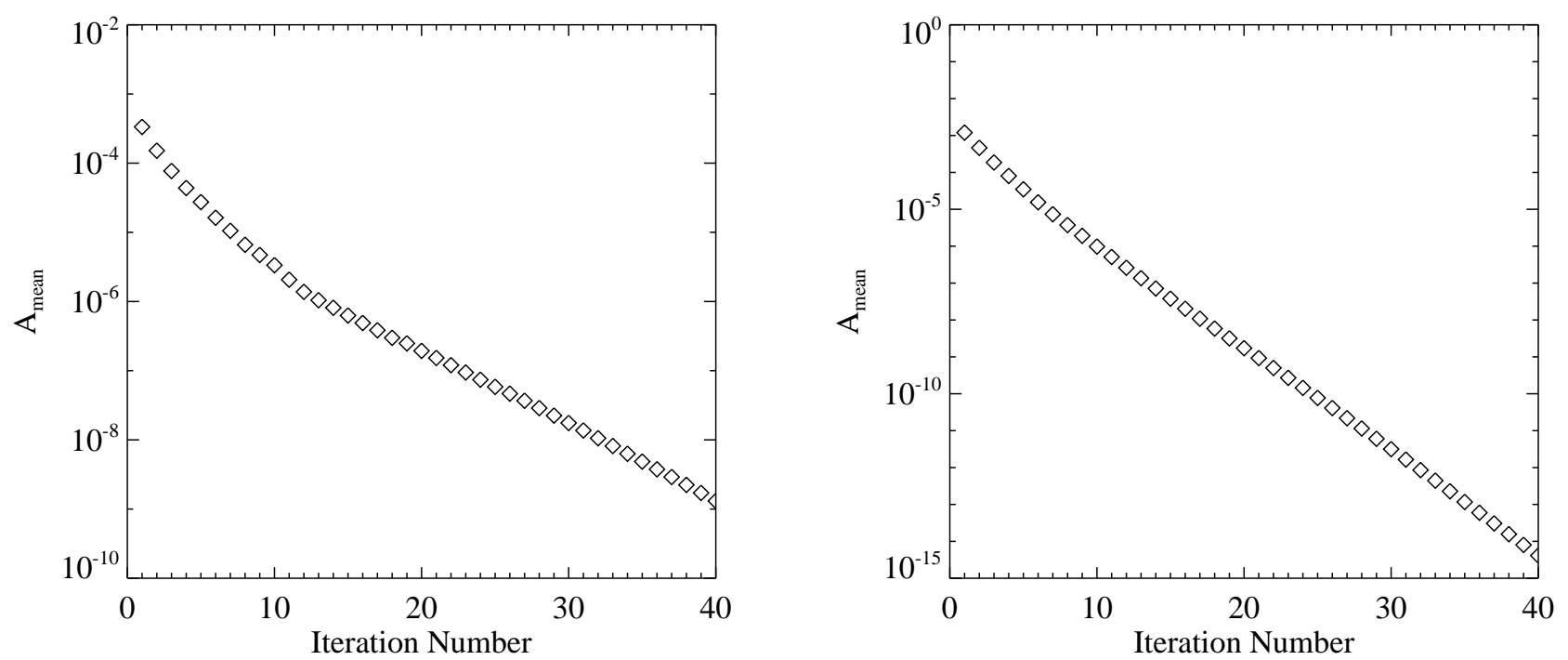

Figure 2. The average absolute change in the field, $A_{\text {mean }}$, as a function of iteration number for the first test case (left panel) and the second test case (right panel). The vertical scale is logarithmic. 

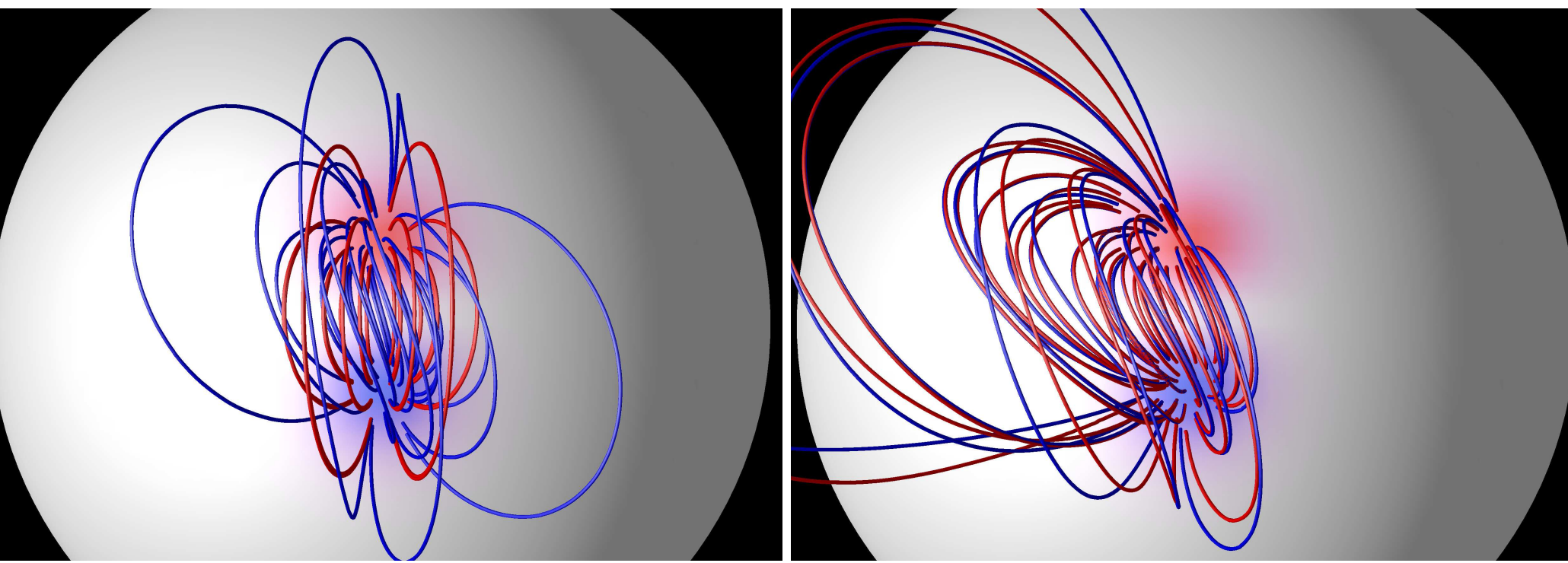

Figure 3. Field lines (blue) and streamlines of current density (red) for test case one. The left panel is after the first Grad-Rubin iteration. The right is after 40 Grad-Rubin iterations. In the left panel there is a clear difference between the two sets of lines, and in the right the two sets of lines coincide, indicating that a force-free solution has been found. The discrepancy between the two sets of lines is due to the interpolation of field values between grid points necessary to draw the lines (see the discussion in Section 4.3.
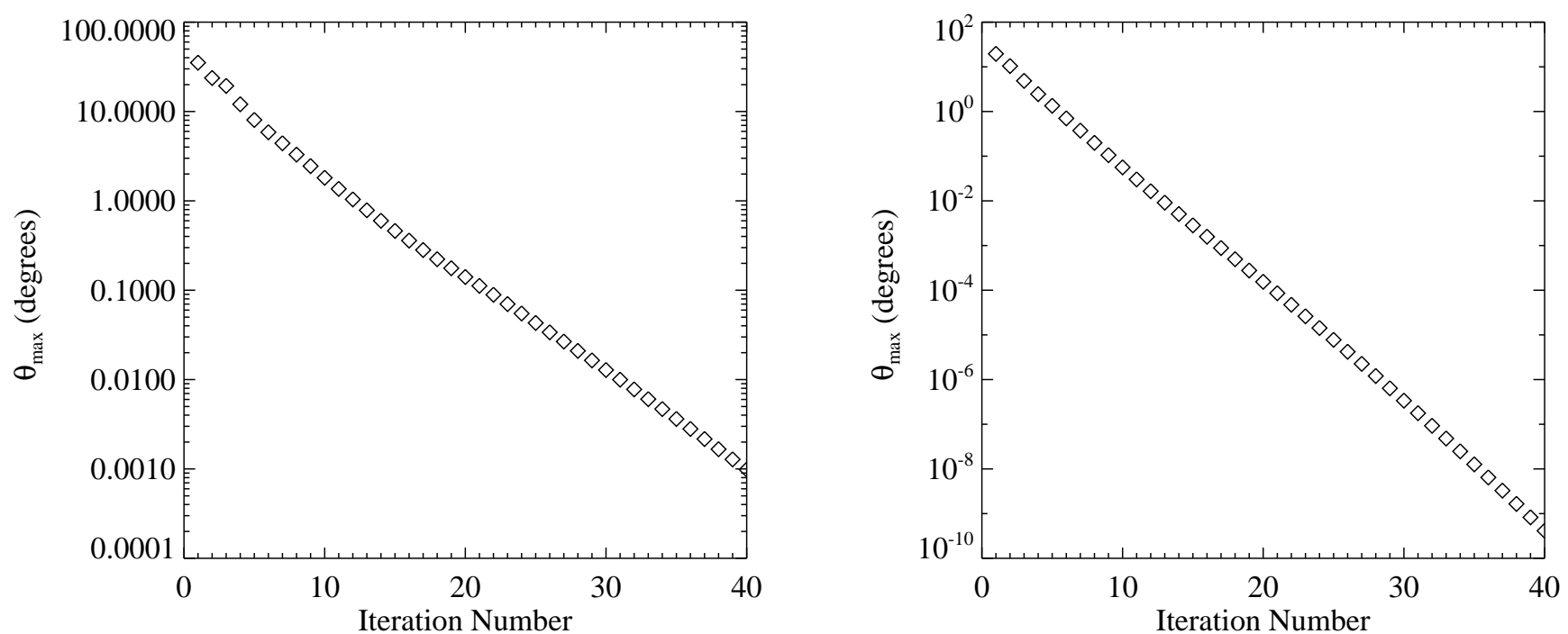

Figure 4. The maximum angle between $\mathbf{J}$ and $\mathbf{B}$ over the solution domain as a function of iteration number. The left panel shows $\theta_{\max }$ for the first test case in $\Omega_{\text {global }}$, and the right panel shows $\theta_{\max }$ for the second test case in $\Omega_{\text {wedge }}$. The vertical scale is logarithmic. 

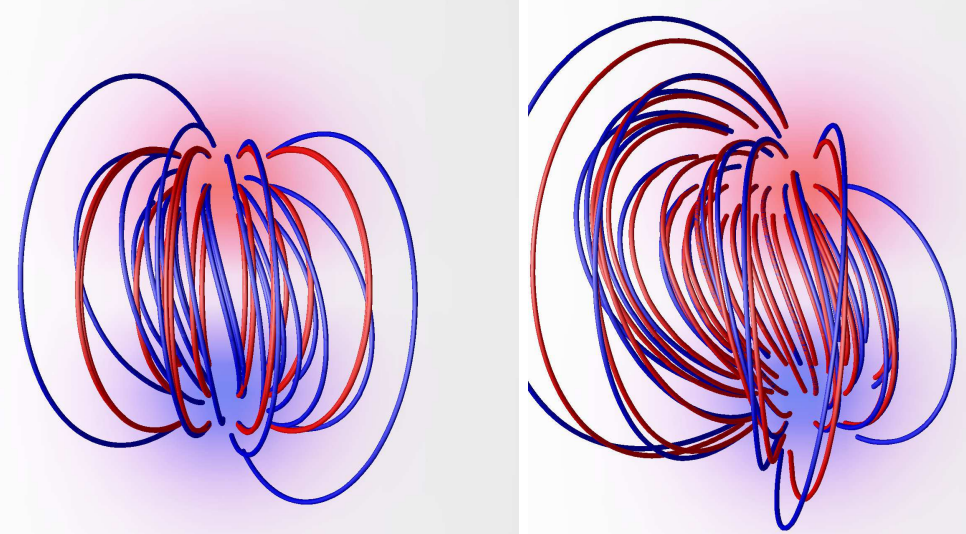

Figure 5. Field lines (blue) and streamlines of current density (red) for test case two. The left panel is after the first Grad-Rubin iteration. The right is after 40 Grad-Rubin iterations. In the left panel there is a clear difference between the two sets of lines, and in the right the two sets of lines coincide, indicating that a force-free solution has been found. The discrepancy between the two sets of lines is due to the interpolation of field values between grid points necessary to draw the lines (see the discussion in Section 4.3). 
Acknowledgements S. A. Gilchrist acknowledges the support of an Australian Postgraduate Research Award.

\section{References}

Alissandrakis, C.E.: 1981, Astron. Astrophys. 100, 197.

Altschuler, M.D., Newkirk, G.: 1969, Solar Phys. 9, 131. doi:10.1007/BF00145734.

Amari, T., Boulmezaoud, T.Z., Mikic, Z.: 1999, Astron. Astrophys. 350, 1051.

Amari, T., Aly, J.J., Canou, A., Mikic, Z.: 2013, Astron. Astrophys. 553, A43. doi:10.1051/0004-6361/201220787.

Barrera, R.G., Estévez, G.A., Giraldo, J.: 1985, Eur. J. Phys. 6, 287.

Boyd, J.P.: 2001, Chebyshev and Fourier Spectral Methods, 2nd edn. Dover Publications Inc, New York, 380.

Chandra, R., Menon, R., Dagum, L., Kohr, D., Maydan, D., McDonald, J.: 2001, Parallel Programming in OpenMP, Morgan Kaufmann Publishers, San Francisco, 1.

De Rosa, M.L., Schrijver, C.J., Barnes, G., Leka, K.D., Lites, B.W., Aschwanden, M.J., et al.: 2009, Astrophys. J. 696, 1780. doi:10.1088/0004-637X/696/2/1780.

Dennis, S.R., Quartapelle, L.: 1985, J. Comp. Phys. 61, 218.

Gary, G.A.: 2001, Solar Phys. 203, 71.

Gary, G.A., Hagyard, M.J.: 1990, Solar Phys. 126, 21.

Grad, H., Rubin, H.: 1958, Proc. 2nd Int. Conf. Peaceful Uses of Atomic Energy, 31, 190.

Jackson, J.D.: 1998, Classical Electrodynamics, 3rd edn. Wiley, New York, 180.

Landi Degl'Innocenti, E., Landolfi, M.: 2004, Polarization in Spectral Lines, Kluwer Academic Publishers, Dordrecht, 625. 
Low, B.C., Lou, Y.Q.: 1990, Astrophys. J. 352, 343. doi:10.1086/168541.

Metcalf, T.R., Jiao, L., McClymont, A.N., Canfield, R.C., Uitenbroek, H.: 1995, Astrophys. J. 439, 474. doi:10.1086/175188.

Molodensky, M.M.: 1974, Solar Phys. 39, 393. doi:10.1007/BF00162432.

Morse, P.M., Feshbach, H.: 1953, Methods of Theoretical Physics, Part Two, McGrawHill, New York, 1252.

Nakagawa, Y., Raadu, M. A.: 1972, Solar Phys. 25, 127. doi:10.1007/BF00155751.

Pesnell, W.D., Thompson, B.J., Chamberlin, P.C.: 2012, Solar Phys. 275, 3. doi:10.1007/s11207-011-9841-3.

Press, W.H., Teukolsky, S.A., Vetterling, W.T., Flannery, B.P.: 2007, Numerical Recipes. The Art of Scientific Computing, 3rd edn. Cambridge University Press, Cambridge, 292.

Régnier, S. 2009, Astron. Astrophys. 497, 17. doi:10.1051/0004-6361/200811502.

Régnier, S.: 2012, Solar Phys. 277, 131. doi:10.1007/s11207-011-9830-6.

Sakurai, T.: 1981, Solar Phys. 69, 343. doi:10.1007/BF00149999.

Sakurai, T.: 1989, Space Sci. Rev. 51, 11. doi:10.1007/BF00226267.

Schou, J., Scherrer, P.H., Bush, R.I., Wachter, R., Couvidat, S., Rabello-Soares, M.C., et al.: 2012, Solar Phys. 275, 229. doi:10.1007/s11207-011-9842-2.

Schrijver, C.J., De Rosa, M.L., Metcalf, T.R., Barnes, G., Lites, B., Tarbell, T., et al.: 2008, Astrophys. J. 675, 1637. doi:10.1086/527413.

Snir, M., Otto, S., Huss-Lederman, S., Walker, D., Dongarra., J: 1998, MPI-the complete reference, 2nd edn. MIT Press, Massachusetts, 1.

Sturrock, P.A.: 1994, Plasma Physics, An Introduction to the Theory of Astrophysical, Geophysical and Laboratory Plasmas, Cambridge University Press, Cambridge, 206. 
Sun, X., Liu, Y., Hoeksema, J.T., Hayashi, K., Zhao, X.: 2011, Solar Phys. 270, 9. doi:10.1007/s11207-011-9751-4.

Tóth, G., van der Holst, B., Huang, Z.: 2011, Astrophys. J. 732, 102. doi:10.1088/0004$637 \mathrm{X} / 732 / 2 / 102$.

Valori, G., Kliem, B., Keppens, R.: 2005, Astron. Astrophys. 433, 335. doi:10.1051/0004-6361:20042008.

Wheatland, M.S.: 2007, Solar Phys. 245, 251. doi:10.1007/s11207-007-9054-y.

Wheatland, M.S., Régnier, S.: 2009, Astrophys. J. Lett. 700, L88 doi:10.1088/0004$637 \mathrm{X} / 700 / 2 / \mathrm{L} 88$.

Wheatland, M.S., Sturrock, P.A., Roumeliotis, G.: 2000, Astrophys. J. 540, 1150. doi:10.1086/309355.

Wiegelmann, T.: 2004, Solar Phys. 219, 87. doi:10.1023/B:SOLA.0000021799.39465.36.

Wiegelmann, T.: 2007, Solar Phys. 240, 227. doi:10.1007/s11207-006-0266-3.

Wiegelmann, T.: 2008, J. Geophys. Res. 113, 3. doi:10.1029/2007JA012432.

Wiegelmann, T., Sakurai, T.: 2012, Living Rev. Solar Phys. 9, 5. http://www.livingreviews.org//rsp-2012-5

Wu, S.T., Sun, M.T., Chang, H.M., Hagyard, M.J., Gary, G.A.: 1990, Astrophys. J. 362, 698. doi:10.1086/169307.

Zwillinger, D.: 1989, Handbook of Differential Equations, 2nd edn. Academic Press, San Diego, 356 . 